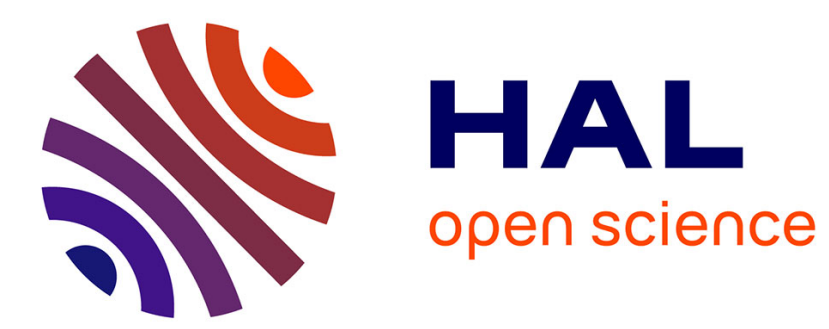

\title{
Ce que sentent les jeunes filles Érika Wicky
}

\section{To cite this version:}

Érika Wicky. Ce que sentent les jeunes filles. Romantisme: la revue du dix-neuvième siècle, 2014, 165 (3), pp.43-53. 10.3917/rom.165.0043 . hal-01626943

\section{HAL Id: hal-01626943 \\ https://hal.univ-rennes2.fr/hal-01626943}

Submitted on 3 Jan 2022

HAL is a multi-disciplinary open access archive for the deposit and dissemination of scientific research documents, whether they are published or not. The documents may come from teaching and research institutions in France or abroad, or from public or private research centers.
L'archive ouverte pluridisciplinaire HAL, est destinée au dépôt et à la diffusion de documents scientifiques de niveau recherche, publiés ou non, émanant des établissements d'enseignement et de recherche français ou étrangers, des laboratoires publics ou privés. 


\title{
CE QUE SENTENT LES JEUNES FILLES
}

\author{
Érika Wicky
}

\section{Armand Colin | Romantisme}

$2014 / 3-n^{\circ} 165$
pages 43 à 53

\section{ISSN 0048-8593}

Article disponible en ligne à l'adresse:

http://www.cairn.info/revue-romantisme-2014-3-page-43.htm

Pour citer cet article :

Wicky Érika, «Ce que sentent les jeunes filles »,

Romantisme, 2014/3 n¹65, p. 43-53. DOI : 10.3917/rom.165.0043

Distribution électronique Cairn.info pour Armand Colin.

(c) Armand Colin. Tous droits réservés pour tous pays.

La reproduction ou représentation de cet article, notamment par photocopie, n'est autorisée que dans les limites des conditions générales d'utilisation du site ou, le cas échéant, des conditions générales de la licence souscrite par votre établissement. Toute autre reproduction ou représentation, en tout ou partie, sous quelque forme et de quelque manière que ce soit, est interdite sauf accord préalable et écrit de l'éditeur, en dehors des cas prévus par la législation en vigueur en France. II est précisé que son stockage dans une base de données est également interdit. 


\section{Érika Wicky}

\section{Ce que sentent les jeunes filles}

Mais si les jeunes filles, si les femmes sont des fleurs

(les poètes le disent [...], l'opinion des maîtres fait loi),

Eh bien ! toute fleur ne doit-elle pas avoir son parfum ?

Jeune fille anonyme citée par Olivier DE TRÉVILLE, Les jeunes filles peintes par elles-mêmes, 1901

Manifestation éphémère et invisible d'une présence matérielle, le parfum se caractérise par son odeur agréable propice à la séduction, à la rêverie et à l'imagination. Il stimule aussi l'éveil des émotions et la constitution des souvenirs en s'adressant au sens le plus intime ${ }^{1}$. Insaisissable, il ne procure en revanche qu'un savoir intuitif, relevant de l'instinct, et s'oppose à toute connaissance objective ${ }^{2}$. Grâce à ces propriétés qui lui étaient alors prêtées, le parfum métaphorise à merveille, au XIX $\mathrm{e}^{\mathrm{e}}$ siècle, la connaissance que la jeune fille se forme d'elle-même et de l'amour, ainsi que, surtout, la façon dont on la conçoit alors. Aussi, le parfum est-il omniprésent lorsqu'il s'agit d'évoquer la jeune fille ou bien de s'adresser à elle ${ }^{3}$. Motivée par la comparaison de la jeune fille avec les fleurs printanières, la métaphore impliquant le parfum est souvent choisie pour évoquer le caractère transitoire de cet état. Parce qu'elle n'est déjà plus petite fille sans être encore femme, la jeune fille se situe dans un énigmatique entre deux social, mais aussi biologique et moral. Cette situation transitoire étant alors perçue comme déterminante pour son aptitude à remplir les fonctions auxquelles on la destine $^{4}$, il apparaît nécessaire de définir la psychologie de la jeune fille, tâche à laquelle le parfum concourt encore, soutenu par la synonymie entre sentir et ressentir. Née de

1. Voir, notamment, à ce sujet : Hippolyte Cloquet, Osphrésiologie ou traité des odeurs, du sens et des organes de l'olfaction, Paris, Méquignon-Marvis, 1821, mais aussi Catherine Schnedecker, « Quand la sémantique se met au parfum », Langages, n 181, 2011. ; Annick Le Guérer, Les Pouvoir de l'odeur, Paris, Odile Jacob, 2002 et Chantal Jaquet, Philosophie de l'odorat, Paris, PUF, 2010.

2. Parmi de nombreux exemples, citons Longet : «Comparé à la vue, au tact, à l'ouie, ces trois sources abondantes de nos sensations et de nos idées, l'odorat apprend peu à l'intelligence » (F.-A. Longet, Traité de physiologie, t. III, Paris, G. Baillière, 1873, p. 47).

3. Sur l'importance de l'olfaction dans le discours tenu sur les femmes, voir le chapitre « The scented womb and the seminal eye : Embodying gender codes through the senses » dans Constance Classen, The Color of Angels : Cosmology, Gender and the Aesthetic Imagination, Londres, Routledge, 1998.

4. Voici une opinion très répandue : «En effet, la jeune fille n'est que la fleur encore à sa naissance de la bonne mère de famille, de la chaste et dévouée épouse, de la femme forte et courageuse qui devra peut-être un jour, elle la frêle créature, soutenir par son courage, sa force morale, sa résignation, son dévouement, toutes les fatigues et les souffrances du foyer » (Comtesse De Bassanville, Julie Fertiault et Céline d'Ornans, La jeune fille chez tous les peuples : études morales, Paris, Amable Rigaud, 1861, p. 6). 
l'avènement de deux valeurs bourgeoises, la famille et l'hygiène $e^{6}$, la jeune fille idéale pourrait être personnifiée par la figure emblématique de Césarine Birotteau, fille du probe parfumeur imaginé par Balzac ${ }^{7}$. Elle s'épanouit pendant toute la seconde moitié du siècle, alors que s'affirme sa place au sein du foyer familial et que la parfumerie, connaissant un formidable essor industriel et commercial, démocratise l'accès aux senteurs. Pendant cette période, l'iconographie ainsi que la littérature, le discours médical, religieux et commercial composent tous ensemble un portrait olfactif de la jeune fille. L'imaginaire déployé alors est renforcé par les usages auxquels invitent les innombrables prescriptions adressées aux jeunes filles par les traités d'hygiène, les manuels de piété et de convenances ainsi que par la réclame. C’est ce portrait olfactif à la chimie complexe qu'il s'agit de dresser pour interroger les marges de l'indéfinissable et de l'indicible dans les conceptions et représentations de la jeune fille et pour préciser les contours de cette nouvelle figure apparue dans l'imaginaire au cours du XIX ${ }^{\mathrm{e}}$ siècle.

\section{MIGNONNE, ALLONS VOIR SI LA ROSE...}

Bien que la comparaison des jeunes filles avec les fleurs se présente déjà, depuis Le Roman de la Rose, comme un des poncifs littéraires les plus ressassés, elle perdure encore dans les textes et les arts visuels du XIX siècle [fig. 1]. Les représentations de jeunes filles en fleurs abondent pendant tout le siècle, fournissant un registre sobre, charmant et asexué pour évoquer la puberté considérée comme le printemps de la vie. Ainsi, dans l'une des pages les plus lyriques de sa thèse de doctorat en médecine, Charles Picard affirme que la femme est « telle que la plante qui, jusqu’à son entier développement, n'a frappé la vue que par l'élévation de sa tige, mais qui, arrivée au terme de sa croissance, n'entrouvre le calice qui recèle les fleurs dont elle tire sa beauté et son principal ornement, et montre tout à coup les organes de la reproduction jusque-là cachés dans son intérieur ${ }^{8}$ ". La métaphore végétale a la faveur des médecins 9 qui convoquent à l'envi l'éclosion, la floraison, l'épanouissement pour décrire non seulement l'évolution des formes des jeunes filles, mais aussi celle de leur odorant sillage.

Le parfum apparaît tantôt comme une cause, tantôt comme une conséquence de l'analogie entre la jeune fille et la plante. Comme y invite cet auteur, "Observons

5. Claudie Bernard, Penser la famille au XIX ${ }^{e}$ siècle, Saint-Étienne, Publications de l'Université de Saint-Étienne, 2007.

6. Alain Corbin, Le miasme et la Jonquille : l'odorat et l'imaginaire social (XVIII $-X I X^{e}$ siècle), Paris, Aubier-Montaigne, 1982.

7. Honoré de Balzac, Histoire de la grandeur et de la décadence de César Birotteau, parfumeur, chevalier de la Légion d'honneur, maire du II arrondissement de la ville de Paris : nouvelle scène de la vie parisienne, Paris, Boulé, 1838.

8. Charles Picaud, Considérations générales sur l'influence de la puberté, de la première menstruation et du mariage sur la santé des jeunes filles, thèse de doctorat, Paris, Didot, 1826.

9. Dans un chapitre intitulé «Le Vœu de la nature », Alain Corbin s'étonne du caractère poétique des pages que les médecins consacrent à la puberté des jeunes filles alors qu'ils mentionnent à peine celle des garçons (Alain Corbin, L'Harmonie des plaisirs : les manières de jouir du siècle des Lumières à l'avènement de la sexologie, Paris, Perrin, 2007). 


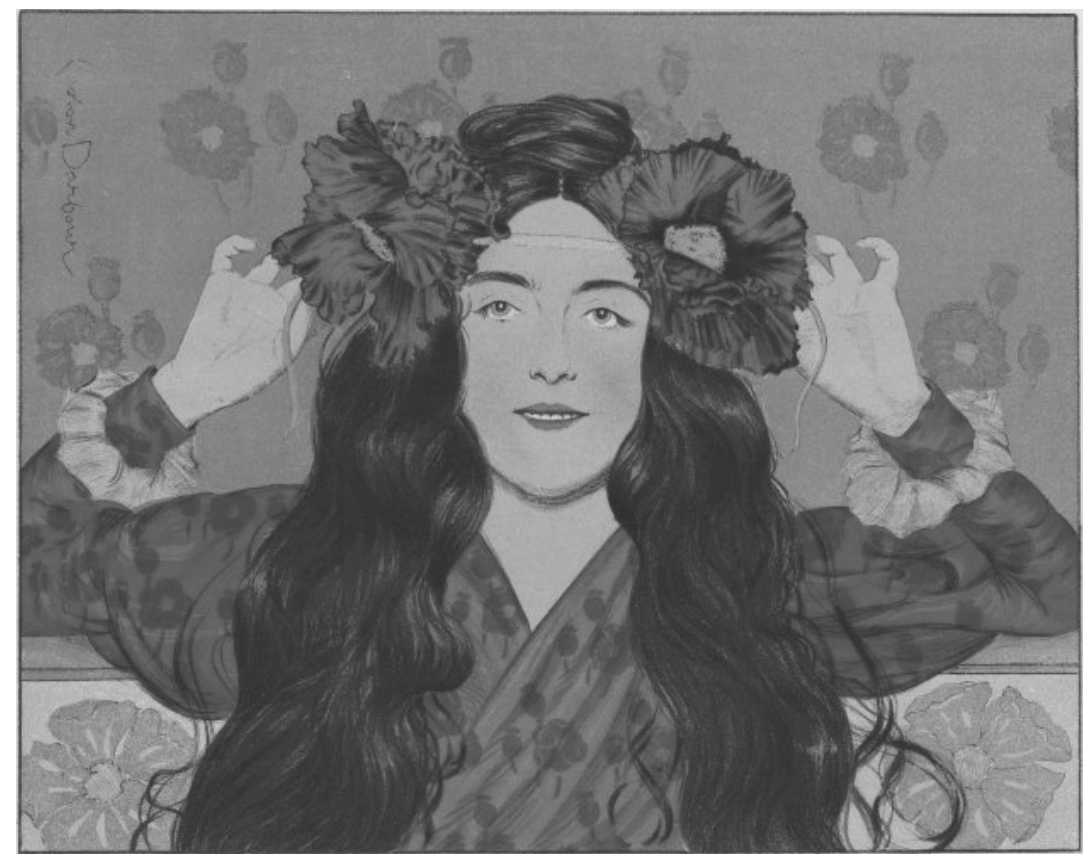

Figure 1. Gaston Darbour, Jeune fille aux coquelicots, L'Estampe Moderne, lithographie couleur, 26 $\mathrm{cm} \times 33 \mathrm{~cm}, 1898$. CBritish Museum

que la jeune fille, ainsi que la jeune plante, exhale cette émanation embaumée : l'une a sa puberté, l'autre sa floraison ${ }^{10}$ ". Véritable topos, le parfum des jeunes filles relève de l'évidence, son constat est souvent réitéré avec la même assurance que celle que montre ici le Dr Fauconney : "Il est certain que la jeune fille vierge est sensiblement aromatique, elle dégage des odeurs printanières ${ }^{11}$. " Pourtant l'énonciation de cette certitude a de quoi surprendre, car contrairement à leurs rondeurs, l'odeur des jeunes filles est impossible à observer ou à mesurer ${ }^{12}$. De telles remarques constituent donc une entorse considérable à la doxa médicale de l'époque qui, fondant notamment l'objectivité scientifique sur la mesure et la quantification des observations ${ }^{13}$, avait depuis longtemps abandonné l'usage de l'olfaction dans la sémiologie médicale ${ }^{14}$.

Quel est donc ce parfum qui s'impose avec tant d'évidence aux narines des savants? Comment a-t-il pu les troubler au point de les aveugler? Un auteur

10. Emmanuel-Napoléon Santini de Riols, Les parfums magiques, Paris, L. Genonceaux, 1903.

11. Jean Fauconney, La Volupté et les Parfums; rapport des odeurs avec le sens génital, le parfum naturel de la femme, Paris, C. Offenstadt, 1903.

12. Au sujet des tentatives allant dans ce sens, voir : Charles Henry, Les odeurs : démonstrations pratiques avec l'olfactomètre et le pèse-vapeur, Paris, Hermann, 1892.

13. Voir : Nélia Dias, La mesure des sens : les anthropologues et le corps humain au XIXe siècle, Paris, Aubier, 2004 ainsi que Lorraine Daston et Peter Gallison, « The Image of Objectivity », Representations, $\mathrm{n}^{\circ} 40,1992$, p. $81-128$.

14. Voir à ce sujet les regrets formulés dans : Augustin Jacob Landré-Beauvais, Séméiotique, ou traité des signes des maladies, Paris, J. A. Brosson, 1818 ( $3^{\mathrm{e}}$ édition), p. 372. 
particulièrement sensible aux parfums, Maupassant ${ }^{15}$, nous procure une hypothèse à travers les interrogations de Servigny, répondant au surnom de Muscade :

Elle sentait bon, sans qu'on pût déterminer quelle odeur vague et légère voltigeait autour d'elle. Ce n'était pas un des lourds parfums de sa mère, mais un souffle discret où il croyait saisir un soupçon de poudre d'iris, peut-être aussi un peu de verveine. D'où venait cette senteur insaisissable ? De la robe, des cheveux ou de la peau ? Il se demandait cela, et comme elle lui parlait de très près, il recevait en plein visage son haleine fraîche qui lui semblait aussi délicieuse à respirer. Alors il pensa que ce fuyant parfum qu'il cherchait à reconnaître n'existait peut-être qu'évoqué par ses yeux charmés et n'était qu'une sorte d'émanation trompeuse de cette grâce jeune et séduisante ${ }^{16}$.

Ainsi perçu, le parfum de la jeune fille semble doté d'une puissance magique, il métaphorise le charme, au sens fort, insaisissable de la jeunesse, qui fait tourner les têtes. Bien que l'odeur soit toujours odeur de ce qui l'exhale, la jeune fille désirable, comme par magie, ne sent pas la jeune fille, mais plutôt les fleurs printanières.

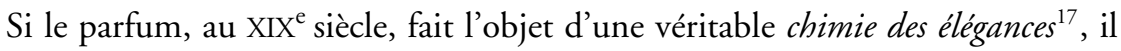
occupe aussi une place prépondérante dans la chimie amoureuse. L'enjeu est tel qu'il figure parmi les préceptes énoncés dans l'opuscule Ce qu'une jeune fille doit savoir avant de se marier: "Qu'il est vrai pourtant que le parfum qu' exhale une femme donne une griserie, une attirance magnétique qui tiennent de la féerie et auxquelles nul n'échappe. Le choix d'un parfum est le pivot du succès de l'élégance et la clef de son charme ${ }^{18}$."Constaté par les médecins ${ }^{19}$, l'ensorcellement provoqué par le parfum de la jeune fille l'est aussi par les romanciers qui en font un de leurs ressorts favoris. Véritable moteur du récit dans le roman érotique Odor di femina ${ }^{20}$, le parfum de la jeune fille doit le plus souvent son efficacité romanesque aux revirements qu'il provoque en s'opposant inopinément aux choix de la raison. Ainsi, le héros du Roman d'une jeune fille pauvre d'Hector France se méfie-t-il d'Angèle, critiquant au passage l'éducation traditionnellement donnée aux jeunes filles :

Quelque chose lui disait que cette jolie tête était vide, son caractère futile et vain, que c'était une de ces gracieuses poupées comme il s'en fabrique dans les riches pensionnats, vernissées d'une légère couche de toutes sciences, sachant dessiner, pianoter, chanter, broder, ce que dans le monde on appelle les jeunes filles parfaites, mais incapables comme épouses de faire la joie d'un foyer.

15. Voir Jean-Alexandre Perras et Érika Wicky, « La sémiologie des odeurs au XIX ${ }^{\mathrm{e}}$ siècle : du savoir médical à la norme sociale », Études françaises, vol. 49, n³ 3, automne 2013.

16. Guy de Maupassant, Yvette, Paris, Victor Havard, 1885.

17. Eugénie Briot, thèse de doctorat, La chimie des élégances : La parfumerie parisienne au XIXe siècle, naissance d'une industrie du luxe, Conservatoire national des arts et métiers, 2008.

18. Ce qu'une jeune fille doit savoir avant de se marier, Nantes, Brelet et Beaussier, sans date. On ne compte pas, par ailleurs, les héroïnes de roman ayant exploité cette ressource du parfum pour séduire.

19. Le Dr Galopin constate : «Aucun sens n'agit plus directement sur la pulpe cérébrale que le sens olfactif. [...] Aucun amant, aucun époux ne résiste à la tentation, aux provocations et aux caresses d'une femme aimée, dégageant, dans l'ombre, un parfum naturel enivrant et suave » (Augustin Galopin, $L e$ parfum de la femme et le sens olfactif dans l'amour, étude psycho-physiologique, Paris, E. Dentu, 1886, p. 103).

20. E.D., Odor di femina : Amours naturalistes, Lebaucher, Montréal, ca 1919 [1900]. 
Plutôt qu'un chef-d'œuvre des arts décoratifs, Olivier recherche une épouse élégante, une femme du monde susceptible de lui ouvrir les portes des salons auxquels il prétend accéder. Vaines ambitions. Il se révèle incapable de résister au sillage de la jeune fille : "Assis à côté d'Angèle, il respirait le parfum qui s'exhalait de la jeune fille, s'en grisait, et de violents désirs s'emparaient de lui ${ }^{21}$.» On devine que ce parfum changera le cours du récit.

\section{SENT-BON ET MAUVAISES LECTURES}

L'érotisation olfactive de la jeune fille trouve son pendant dans l'évocation de la jeune fille respirant le parfum des fleurs. À la faveur d'un glissement opéré parmi les trois acceptions du verbe sentir (exhaler, flairer, ressentir), la jeune fille qui émeut les sens parce qu'elle sent les fleurs, se trouve elle-même troublée lorsqu'elle sent des fleurs ${ }^{22}$. Cet imaginaire est particulièrement manifeste dans l'iconographie de l'époque ${ }^{23}$ [fig. 2] où l'olfaction est toujours présentée, à l'instar de la lecture, comme une activité solitaire et sensuelle, propice à toutes les rêveries. S'il lui est permis d'embaumer sans le savoir, respirer le parfum des fleurs parait être un geste suspect pour la jeune fille à une époque où la principale attente nourrie à l'égard des jeunes filles est l'absence de désir ${ }^{24}$. Cette défiance donne lieu à des scènes telles que celle placée par René Boylesve dans le récit de sa narratrice :

Je me tenais volontiers assise près de mon balcon, au-dessus de l'œil sombre de la citerne, mon bras nu appuyé sur la rampe de fer froid, et la bouche suçant comme un fruit le dessus de ma main. L'air, à peine agité, apportait par moments un parfum mêlé d'héliotropes et de framboises auquel se joignait l'odeur de futailles qui imprègne le pays à l'approche des vendanges. Mon Dieu ! mon Dieu ! qu'avezvous mis en moi à cette époque de ma vie? Quelle puissance de ravissement m'avez-vous donnée à dix-sept ans, que je n'ai plus retrouvée depuis ${ }^{25}$ ?

Ce ravissement qui vient si promptement aux jeunes filles s'explique, selon la science de l'époque, par l'ardeur de leur imagination ${ }^{26}$ qui, au moment de la puberté, remplace la mémoire dans le rôle de faculté maîtresse. Cette imagination débordante leur fait courir un grand danger, notamment en nourrissant l'intuition des jeunes filles qui sentent quelque chose venir ${ }^{27}$. Aussi est-il nécessaire de limiter ce qui stimule leur imagination, à savoir, essentiellement, les romans et les parfums. Associant les

21. Hector France, Roman d'une jeune fille pauvre, Paris, H. Geoffroy, 1896, p. 386 et 180.

22. On rencontre cependant assez peu de jeunes filles troublées par l'odeur de leurs semblables, si ce n'est dans la littérature érotique. Voir, par exemple : Paul Adam, Chair molle : roman naturaliste, Paris, A. Brancart, 1885.

23. Christina Bradstreet, « Wicked with Roses : Floral Feminity and the Erotics of Scent », NineteenthCentury World Wide, vol. 6, n 1, 2007.

24. Voir Yveline Fumat, «La socialisation des filles au XIX ${ }^{\mathrm{e}}$ siècle », Revue française de pédagogie, $\mathrm{n}^{\circ} 52,1980$.

25. René Boylesve, La jeune fille bien élevée, Paris, Fayard, 1909, p. 98.

26. Voir à ce sujet: Augusta Moll-Weiss, Les mères de demain, l'éducation de la jeune fille d'après sa physiologie, Paris, Vigot frères, 1902.

27. Fritz Nies, « La femme-femme et la lecture, un tour d'horizon iconographique », Romantisme, $1985, \mathrm{n}^{\circ} 47$, p. $97-106$. 
deux vices, Zénaïde Fleuriot situe sa critique des mauvais livres dans le registre olfactif. Elle fustige tout d'abord les ouvrages qui font " rêver d'eau de Cologne à ceux qui sentent l'oignon " et achève sur cette mise en garde : "Tout livre est une fleur, un parfum. Amassez dans vos parterres des fleurs vénéneuses, vous empoisonnez votre atmosphère ${ }^{28}$. "Edmond de Goncourt se livre à une analogie similaire, motivée par l'habitude qu'avaient les jeunes filles de garnir leurs livres de fleurs. Il prête à Chérie, cas exemplaire des effets mortiferes de la mauvaise éducation donnée aux jeunes filles, un usage singulier des parfums : "Le livre qu'elle lisait [Paul et Virginie], elle le trempait, elle le plongeait dans des eaux de senteur, et l'histoire d'amour arrivait à son imagination, à ses sens, par des pages toutes mouillées, tout humides de parfums liquéfiés ${ }^{29}$."

Étant tous deux réputés aphrodisiaques ${ }^{30}$, romans et parfums donnent lieu à de nombreux portraits de jeunes filles, lisant ou flairant, qui constituent autant d'allusions à l'onanisme. Le sujet appelle une certaine délicatesse que satisfait l'allusion au parfum, souvent conviée dans l'argumentation destinée à mettre les jeunes filles en garde contre leur propre sensualité. Là encore, les habitudes de Chérie fournissent un excellent exemple de la nature de l'indicible que le parfum permet d'évoquer :

Maintenant, tous les matins, à son premier réveil, la jeune fille se levait et, encore endormie, d'une main cherchait à tâtons, atteignait un vaporisateur, et se mettait à insuffler l'intérieur de son lit de la senteur de l'héliotrope blanc.

Puis, aussitôt, elle se refourrait entre les draps parfumés, prenait soin de les ouvrir le moins possible. Et, la tête enfoncée sous la couverture jusqu'aux yeux, elle prenait une jouissance indicible à se sentir pénétrée, caressée, rafraîchie par l'humidité odorante de la vaporisation dans laquelle il lui semblait, son être encore mal éveillé, à demi s'évanouir, en parfum et en bonne odeur.

À la fin elle se rendormait, trouvant une volupté dans un sommeil où il y avait un peu d'ivresse cérébrale et d'asphyxie ${ }^{31}$.

L'usage du parfum, comme la lecture, doit être soumis à un rigoureux contrôle, sans quoi il risque d'agir comme un poison ${ }^{32}$. Puisque la sensibilité olfactive des jeunes filles entre en correspondance avec le désir masculin suscité par leur parfum, il apparaît aussi crucial de protéger ces dernières de la convoitise des hommes que de leurs propres penchants. Alors que la lecture familiale, à voix haute, par le père, constituait le modèle idéal de lecture ${ }^{33}$, les plaisirs nés de l'olfaction s'avèrent d'autant plus inquiétants qu'ils sont plus difficiles à censurer.

La religion catholique qui, en vertu du Cantique des Cantiques et de son usage ancestral de l'encens, semble bénéficier d'une expertise en matière de parfums, ne

28. Zénaïde Fleuriot, Les mauvais jours : notes d'un bourru sur le siège de Paris, Paris, C. Dillet, 1872 , p. 68 et 165.

29. Edmond de Goncourt, Chérie, Paris, Charpentier, 1884.

30. Martyn Lyons, Readers and Society in Nineteenth-Century France : Workers, Women, Peasants, Basingstoke, Palgrave Macmillan, 2001.

31. Ouvr. cité, p. 302.

32. Ruth Gantert, « Modèles de la lecture féminine à la fin du XIX ${ }^{\mathrm{e}}$ siècle », dans Femmes et livres, Danielle Bajomée, Juliette Dor et Marie-Élisabeth Henneau (dir.), Paris, L'Harmattan, 2007, p. 125-137.

33. Belinda Jack, The Woman Reader, New Haven Yale University Press, 2012. 


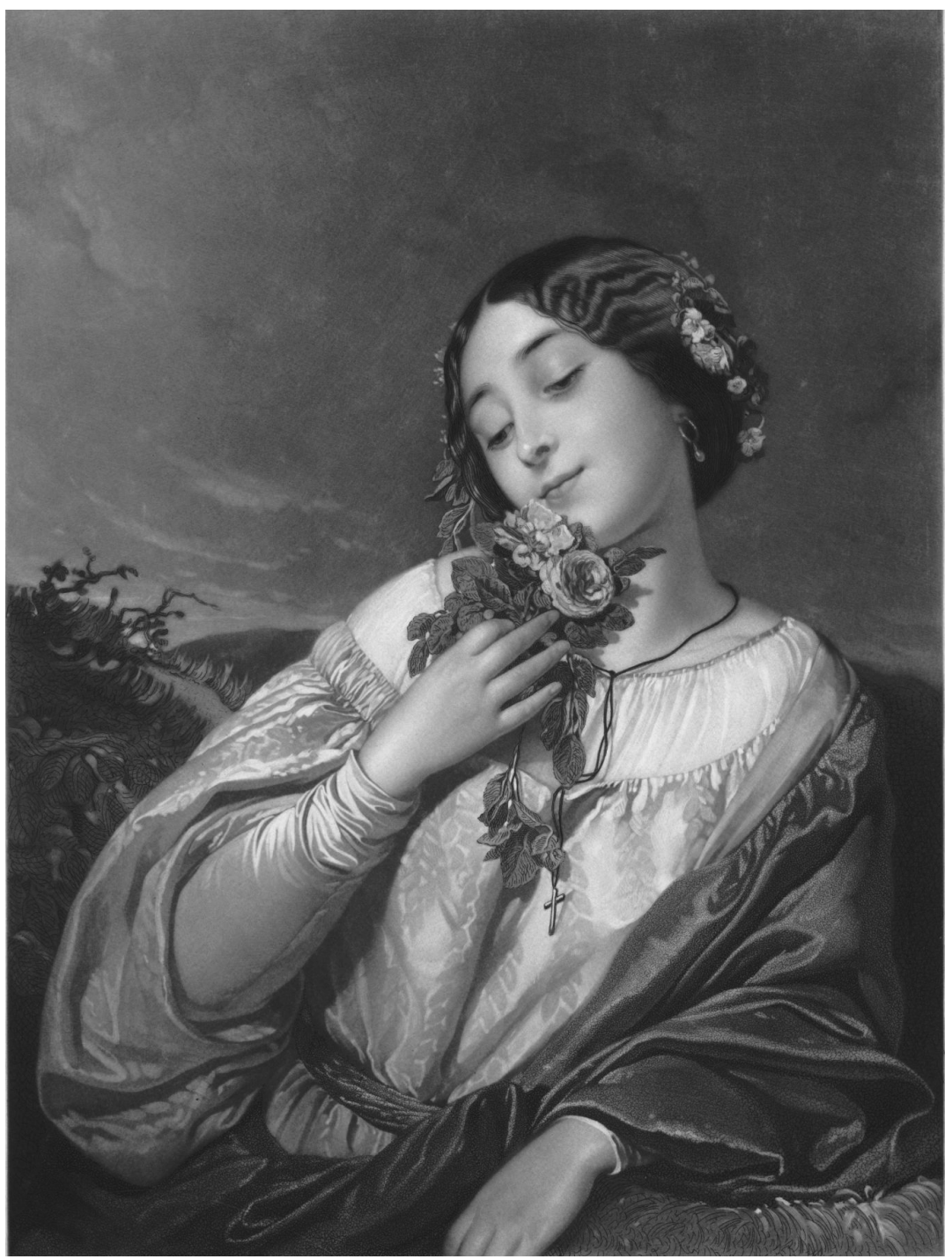

Figure 2. H. Garnier, L'Odorat/Smell, gravure d'après un tableau de Schlesinger, $49 \times 35 \mathrm{~cm}$, Paris/New York, Goupil et Compagnie, 1850. CBritish Museum

manque pas d'aborder ce sujet, et ce en dépit des diatribes anticléricales auxquelles donne souvent lieu la propreté relative des couventines. L'odeur de sainteté ${ }^{34}$, qui se manifeste comme la trace sensible d'une vertu invisible, fournit un succédané spirituel aux séductions de la parfumerie. Elle constitue aussi un modèle pour les

34. Jean-Louis Benoit, « Autour de l'odeur de sainteté : les parfums dans le monde chrétien », Iris, $n^{\circ} 33,2012$. 
jeunes filles dont les actions doivent dégager un parfum qui, montant au ciel comme une offrande, s'avère bien préférable à celui du bain qui menace leur chasteté.

Des textes comme ce poème de Marc Monnier intitulé Beauté, Bonté invitent donc les jeunes filles à revêtir les parfums floraux de la vertu :

Mais il est un parfum céleste

Plus fécond encore en douceur,

Qui d'une fleur simple et modeste

Peut faire une divine fleur.

Arôme du lis angélique,

Baume de la rose mystique

Par la terre au ciel emprunté,

Comme l'encens des sacrifices,

Il répand de saintes délices...

Et ce parfum, c'est la bonté 35 !

Ainsi, l'analogie entre l'odeur et la morale, que l'on a vu à l'œuvre jusqu'à présent, trouve un écho, ou bien une origine, dans le discours religieux. Entre les prescriptions morales des guides de convenance et la dimension spirituelle que lui confere le discours religieux, le parfum acquiert une portée considérable qui ne saurait être envisagée indépendamment de l'essor de l'industrie et du commerce de la parfumerie.

\section{L'ÉCONOMIE OLFACTIVE DE LA JEUNE FILLE : UN ÉQUILIBRE PRÉCAIRE}

Comme pour la sexualité ${ }^{36}$, le bon usage des parfums, dont l'industrie est généreuse au XIX ${ }^{\mathrm{e}}$ siècle, est affaire de poids et de mesure. La jeune fille est le lieu d'un équilibre olfactif délicat. Alors qu'elle embaume les fleurs des champs, déjà la puberté, s'accompagnant des « exhalations fétides ${ }^{37}$ " des premières règles, fait pencher la balance du côté de la chair. D'autres facteurs peuvent contribuer à déstabiliser cette harmonie olfactive : «La jeune fille, dont les sens n’ont pas encore parlé, est sensiblement aromatique ; elle sent le vent et le soleil du printemps, l'eau fraîche framboisée. Celle qui est amoureuse a un parfum plus prononcé ; celle qui pratique l'onanisme, ou a un amant, rentre dans la catégorie générale ${ }^{38}$. "Selon ce point de vue, l'odeur de la jeune fille est moins influencée par les transformations qui affectent son corps que par celles qui affectent ses sens. Alors que Maupassant évoquait le parfum de la jeune fille comme une construction du désir masculin, il apparaît ici garanti par l'absence de désir et d'expression de soi.

35. Clarisse Juranville, Le Savoir-faire et le savoir-vivre dans les diverses circonstances de la vie, Paris, Boyer, 1879, p. 29.

36. Alain Corbin, ouvr. cité, p. 37-96.

37. Voir à ce sujet: Jean-Yves Le Naour et Catherine Valenti, « Du Sang et des femmes. Histoire médicale de la menstruation à la Belle Époque », Clio, $\mathrm{n}^{\circ}$ 14, 2001.

38. Augustin Galopin, ouvr. cité p. 195 
Seul le mariage et l'acte sexuel qui l'entérine peuvent, en transformant la jeune fille en femme, faire basculer sans déshonneur cet équilibre du floral vers le charnel ${ }^{39}$. Avant cela, la jeune fille doit garder son parfum. En la matière, il ne saurait y avoir de demi-mesure, comme le montre le scandale provoqué par le roman de Marcel Prévost Les Demi-Vierges ${ }^{40}$. Or, préserver un parfum est une tâche bien malaisée, car si le parfum n'a pas son pareil pour stimuler la mémoire et créer des souvenirs, il demeure, étymologiquement, ce qui part en fumée. Il est impossible à conserver à moins qu'on ne le garde dans un flacon, comme l'explique un prêtre dans ces lignes très suggestives : « Le parfum se conserve suave et odorant dans l'urne qui le renferme, il perd son odeur si le cristal se brise et le laisse s'épancher au dehors; la fleur se garde fraîche, veloutée à l'abri de la haie épineuse qui la protège ; elle se fane, touchée par la main de ceux qui l'admirent ${ }^{41}$. "

Régler l'usage que les jeunes filles font des senteurs constitue donc, à plusieurs égards, un enjeu majeur. Tout d'abord réprouvé, l'usage de parfums par les jeunes filles s'impose à la fin du siècle. Certes, certains auteurs maintiennent l'interdit et recommandent aux jeunes filles de s'en tenir à la plus stricte hygiène, mais la plupart d'entre eux prennent acte de la mode et autorisent l'usage d'un peu de parfum. Cela semble inévitable compte tenu de l'ambiguïté entre propreté et parfum ménagée par la prolifération de savons parfumés. Cependant, le chapitre du parfum s'accompagne toujours d'un éloge de la juste mesure, tel que celui-ci :

Moins on emploie de parfums, mieux cela convient. Cependant, un soupçon d'odeur très légère et très comme il faut peut être toléré, mais, je le répète, à la condition de rester un soupçon. Un peu de lait d'iris ou d'eau de Cologne dans l'eau dont vous vous servez, c'est parfait. Une goutte d'essence de violette dans votre mouchoir, c'est bien ; mais pas plus, et surtout, bornez-vous à des parfums discrets, qui ne peuvent être antipathiques, ni malfaisants ${ }^{42}$.

C'est de concert que les règles de convenances et celles de l'hygiénisme gouvernent l'usage des parfums. L'excès de parfum étant jugé dangereux pour la santét ${ }^{43}$, limiter son emploi et la fréquentation des bals dont les parfums alourdissent l'atmosphère permet commodément de préserver la discrétion et la modestie qui sied à la jeune fille. Les mêmes motivations ajoutées à la crainte du confinement ${ }^{44}$ justifient que l'on privilégie les senteurs légères aux parfums lourds et capiteux. Quant aux parfums de

39. Zola évoque ainsi « cette odeur humaine, pénétrante, sensuelle, cette odeur d'amour qui s'échappe au matin de la chambre close de jeunes époux » (Zola, La Curée, Paris, Charpentier, 1895).

40. Marcel Prévost, Les Demi-Vierges, Paris, Alphonse Lemerre, 1894. Voir aussi Victor Joze, La ménagerie sociale : le demi-monde des jeunes filles, Paris, Anthony et Compagnie, 1895.

41. Trophime Roucau, Chemin d'ombre, causeries : aux jeunes filles, Paris, P. Lethielleux, 1905.

42. M. Maryan et Gabrielle Béal, Le fond et la forme : le savoir-vivre pour les jeunes filles, Paris, Bloud et Barral, 1896

43. « À petites doses, les odeurs produisent l'exhilaration, un excès d'acuité des sens et de l'imagination ; à doses plus fortes, des troubles de la sensibilité, de l'intelligence et du mouvement » (Paul Sapiens, L'Hygiène dans la famille, Paris, Kolb, 1890). On ne s'étendra pas ici sur tous les troubles que l'on prête à l'usage maladif des parfums, ils sont fort nombreux, voir à ce sujet : Madame Brisset de Nos, Les Jeunes Filles et les Jeunes Femmes, Paris, Perisse Frères, 1862.

44. Coriveaud, Hygiène de la jeune fille, Paris, Baillière, 1882. On ne cessait de recommander le grand air à ces futures femmes d'intérieur, comme le dit la jeune fille de la chanson : « J'aime à rêver sous les 
synthèse produits à moindre coût par l'industrie chimique dès les années 1860 , ils étaient dénigrés au profit de parfums naturels plus conformes à la conception de la jeune fille. Enfin, la simplicité clôt la liste des exigences présentées aux jeunes filles en matière de parfum. En effet, les parfums composés à partir d'essences naturelles sont chers et évoquent un goût du luxe qui augure mal pour une jeune fille à marier, car rappelons-le, c'est pour cela que la jeune fille doit tant plaire. Le parfum idéal de la jeune fille doit donc être léger, floral et naturel autant de caractéristiques qui mettent en évidence l'accord parfait entre le discours médical et littéraire sur le parfum de la jeune fille et les prescriptions qui sont adressées à cette dernière. Justifiant pleinement le choix du mot parfum pour évoquer l'odeur corporelle des jeunes filles, l'industrie de la parfumerie complète cette redondance en offrant aux jeunes filles des senteurs similaires à celles que l'on prête à leur odeur corporelle.

De même, la différence entre le parfum de la jeune fille et celui de la femme se trouve transposée en parfumerie. Comme l'énonce Ernest Monin : «La violette et la lavande ambrée, parfums doux et discrets, conviennent aux jeunes filles, le foin coupé, la peau d'Espagne, plus capiteux, aux femmes faites ${ }^{45}$. "Si les femmes mûres bénéficient d'une plus grande liberté de choix, l'usage qu'elles font des parfums est sensiblement le même que celui que l'on préconise aux jeunes filles [fig. 3]. En effet, les senteurs d'origine animale (musc, civette, etc.) faisant, en parfumerie, l'objet d'une réprobation unanime ${ }^{46}$, les fragrances florales gardaient la faveur des femmes. L'opposition olfactive entre la femme et la jeune fille semble donc aussi relever de la construction discursive. Les catégories végétal/animal et naturel/artificiel, empruntées à la parfumerie, redoublent olfactivement l'opposition entre jeune fille et femme, entre innocence et expérience, articulée autour de l'axe du mariage.

Caractérisé par sa redondance, le discours sur la jeune fille produit pendant la seconde moitié du XIX ${ }^{\mathrm{e}}$ siècle est saturé de senteurs. Le parfum se révèle doté d'une grande propension à évoquer la sensualité de la jeune fille ainsi que son corps et les désirs qu'il suscite, autant de charmes habituellement cantonnés dans le domaine du non-dit ${ }^{47}$. En revanche, lorsque l'on s'adresse à elle, le parfum fait l'objet d'un contrôle, il est convoqué pour réprimer l'expression du corps et des sens. Le portrait olfactif de la jeune fille qui se dessine à travers les écrits qui la concernent accorde une place prépondérante à la virginité, de sorte que c'est la nuit de noce qui, bien plus que la puberté, transforme irrémédiablement l'odeur naturelle de la jeune fille devenue femme. Précieux, fragile, éphémère, susceptible de disparaître ou de se corrompre si

bosquets en fleur, j'aime l'émail, le parfum des campagnes...» Un cœur de jeune fille, paroles de Crevel de Charlemeagne, Musique de Joseph Vimeux.

45. Ernest Monin, L'Hygiène de la beauté : formulaire cosmétique, Paris, Octave Douin, 1890. Voir aussi A. Debay, Les parfums et les fleurs, Paris, Moquet, 1846.

46. Voir Élisabeth-Félicie Bayle-Mouillard, née Élisabeth Canard, dite Celnart, Manuel des dames, ou l'art de l'élégance sous le rapport de la toilette, des honneurs de la maison, des plaisirs, des occupations agréables, Paris, Librairie encyclopédie Roret, 1833 ( $2^{\mathrm{e}}$ éd.), p. 11.

47. Voir Hannah Thompson, Taboo: Corporeal Secrets in Nineteenth-Century France, Londres, Legenda, 2013. 


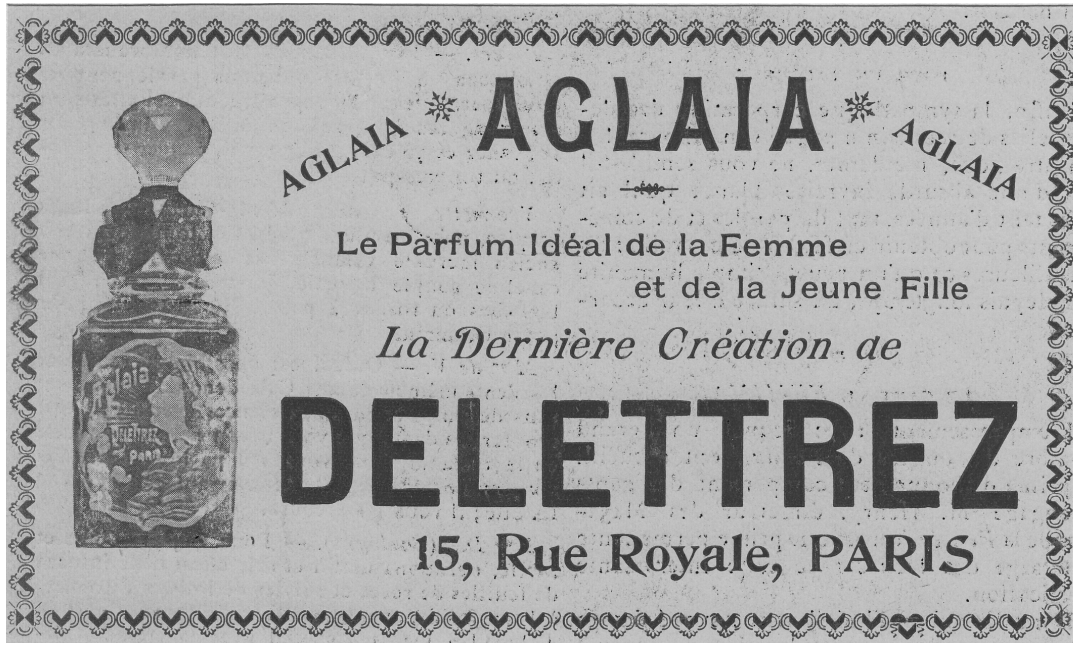

Figure 3. Publicité, $11 \times 8 \mathrm{~cm}, 1902$.

on ne le surveille pas assez étroitement, le parfum de la jeune fille garantit sa valeur d'échange sur le marché du mariage. Il contribue ainsi efficacement à construire la jeune fille à la fois comme objet de fantasme et comme future épouse entièrement dévouée à ses fonctions sociales. Bien qu'elle soit présentée comme naturelle et florale jusque dans les effluves qu'elle dégage, la jeune fille idéale paraît être le fruit d'un imaginaire culturel, un modèle fourni aux jeunes filles dans toute leur diversité olfactive et sociale. Voilà ce qui explique l'absence de voix de jeunes filles ${ }^{48}$ dans l'intense polyphonie qui s'élève autour d'elles à partir du Second Empire, au moment même l'on ne cesse de s'exclamer: Il n'y a plus de jeunes filles ${ }^{49}$ !

(UQAM)

48. À l'exception des manuels de civilité, les textes commentés sont tous rédigés par des hommes. Si certains d'entre eux, comme Edmond Goncourt ou Olivier de Tréville, qui dit avoir interrogé des milliers de jeunes filles, fondent leurs écrits sur des témoignages, ceux-ci corroborent le discours ambiant et ne font pas entendre de voix distincte.

49. Isabelle Bricard, Saintes ou pouliches : l'éducation des jeunes filles au XIXe siècle, Paris, Albin Michel, 1985. 